\title{
La Bibliothèque humaniste de Sélestat en Alsace
}

\author{
MAURICE LEBEL
}

La bibliothèque humaniste de Sélestat, en Alsace, est trop peu connue, même des spécialistes du XVI ${ }^{\mathrm{e}}$ siècle et de la Renaissance. Je ne la connaissais pas moi-même, sauf de nom et de réputation; $\mathrm{j}$ 'avais déjà correspondu, il est vrai, avec son conservateur actuel, M. Hubert Meyer, assisté de Madame Hild, mais c'est seulement en septembre 1976 que j'eus la bonne fortune d'y travailler à loisir. Elle a beau compter plus de cinq siècles d'existence, puisqu'elle date de 1452, rares sont les chercheurs qui la fréquentent au $X X^{\mathrm{e}}$ siècle. Et pourtant des érudits tels que A. Hobson, J. Gény, P. Adam, J. Knepper, A. Dorlan, A. Horawitz et autres ont publié articles de revue, chapitres de volume, biographies, catalogues et ouvrages sur le sujet dans le dessein de faire connaître les trésors artistiques et littéraires qu'elle recèle et qui y sont conservés avec autant de fierté que de soin. Trop d'érudits croient, sans doute à tort, que la Bibliothèque Nationale, à Paris, et celle du British Museum, à Londres, contiennent tous les écrits relatifs à la Renaissance; si uniques soient-elles au monde par leurs richesses extraordinaires, ces deux bibliothèques ne possèdent point cependant plus d'un rare document qui se trouve à Sélestat, comme on peut encore trouver des textes importants en plusieurs bibliothèques privées d'Italie. La bibliothèque humaniste de Sélestat comprend 451 manuscrits, dont près de la moitié sont antérieurs à la fin du $\mathrm{XVI}^{\mathrm{e}}$ siècle, 532 incunables et 1964 imprimés du XVI ${ }^{\mathrm{e}}$ siècle.

M. l'abbé Paul Adam, qui fut de 1952 à 1975 bibliothécaire et archiviste de la ville de Sélestat, éminent historien de l'humanisme des $\mathrm{XV}^{\mathrm{e}}$ et $\mathrm{XVI}^{\mathrm{e}}$ siècles, aussi à l'aise en sociologie religieuse qu'en histoire de l'art et dans les collections les plus célèbres de l'Italie, a écrit plusieurs livres, entre autres, cette étude exemplaire, préfacée par Gabriel Le Bras, La vie paroissiale en France au $X I V^{e}$ siècle, et cet essai, qui a connu trois éditions depuis 1962, L'Humanisme à Sélestat (Sélestat, Imprimerie Alsatia, 1962). Le lecteur y trouvera à la fin de la lère édition, aujourd'hui épuisée, un catalogue sommaire des manuscrits pieusement conservés à la bibliothèque. Le prédécesseur de l'abbé Adam, le chanoine Joseph Walter, archéologue et bibliothécaire bien connu, a consacré la majeure partie de sa vie à la 


\section{2 / Renaissance and Reformation}

composition de ce trésor d'érudition et de science qu'est le Catalogue des incunables et imprimés du XVI siècle (Colmar, 1929). Ouvrage unique, indispensable, un modèle du genre. Ajoutons que la bibliothèque humaniste de Sélestat, logée au $1^{\mathrm{er}}$ étage de la Halle-aux-Blés, est un joyau de la ville.

Sélestat est une belle ville d'art, qui compte depuis 1975 douze siècles d'histoire et dont la population s'élève aujourd'hui à environ 16.000 âmes. Située au coeur de l'Alsace, à $22 \mathrm{~km}$. de Colmar et à $45 \mathrm{~km}$. de Strasbourg, elle occupe une situation médiane, joue, pour ainsi dire, le rôle de trait d'union entre la basse et la haute Alsace. En 775, Charlemagne, alors qu'il était en route pour l'Italie, y séjourna pour parrainer la nouvelle agglomération née sur les bords de l'Ill en célébrant la Noël dans son domaine impérial de Scladdistavi villa. Le Vieux-Sélestat, avec ses ruelles, chargées de passé, pittoresques et sinueuses, comme un merveilleux labyrinthe, ses trois magnifiques églises gothiques et romanes des $\mathrm{XII}^{\mathrm{e}}, \mathrm{XIII}^{\mathrm{e}}$ et $\mathrm{XV}^{\mathrm{e}}$ siècles, ne manque jamais de retenir le visiteur, le touriste peu pressé, qui aime $y$ flâner et rêver à son aise, s'arrêter même dans un caveau pour y déguster une bouteille de vin blanc d'Alsace et une tarte à l'oignon. Un véritable havre de paix et de tranquillité que Sélestat, où il fait bon contempler les maisons médiévales, les armoiries professionnelles, les vieux colombages, les pierres séculaires, les vestiges des remparts des $\mathrm{XIV}^{\mathrm{e}}$ et $\mathrm{XVII}^{\mathrm{e}}$ siècles, la porte dite de Strasbourg, les vieux bastions des Capucins et des Suédois réunis par une courtine, les tours romanes, les dentelles et les flèches gothiques. Et que de fleurs multicolores disséminées un peu partout aux fenêtres des maisons, sur la place, le long des rues, à la gare ferroviaire, d'une propreté exemplaire! On dirait une ville-jardin. Une joie sans pareille pour l'oeil et l'esprit.

L'histoire de Sélestat est étroitement liée à celle du livre. N'y trouve-t-on pas le plus vieux manuscrit d'Alsace, un lectionnaire mérovingien datant de la fin du VII ${ }^{\mathrm{e}}$ siècle? Quant aux manuscrits grecs et latins, ils ont été écrits du IX $\mathrm{X}^{\mathrm{e}}$ au $\mathrm{XV}^{\mathrm{e}}$ siècle inclusivement. Toute l'histoire du livre, on peut la voir se dérouler et en suivre les principales étapes en étudiant les vitrines de la bibliothèque-musée. Elle est aussi liée au livre des miracles de sainte Foy, le fameux Liber Miraculorum Sanctae Fidi, écrit au $\mathrm{XI}^{\mathrm{e}}$ siècle par un moine bénédictin de Conques qui le ramena à Sélestat, où les disciples de saint Benoît fondèrent un monastère en 1094. On peut le tenir pour l'un des trésors de la Bibliothèque. Dans le champ supérieur d'une magnifique miniature extraite du Livre, on voit la jeune martyre, sainte Foy, revêtue du riche costume des dames du $\mathrm{XI}^{\mathrm{e}}$ siècle, suivie de Saint Caprais, évêque d'Agen, qui trouva la mort au même moment qu'elle. Dans le champ inférieur, l'auteur inconnu du Liber Miraculorum supplie humblement la sainte de vouloir bien accepter le manuscrit qu'il lui tend.

On peut voir aussi, dans une niche de la belle et grande salle de lecture, le buste de celle qu'on appelle communément, faute de précision, la belle dame inconnue de Sainte-Foy, dont le sourire énigmatique intrigue tout 
ensemble les historiens et les visiteurs, les archéologues et les artistes. De qui s'agit-il au juste? Ce masque mortuaire, d'une rare beauté empreinte de sérénité devant la mort, reproduit fidèlement un buste féminin à partir d'un bloc de mortier trouvé dans un tombeau lors des travaux de restauration effectués au XIX ${ }^{\mathrm{e}}$ siècle dans l'église Sainte-Foy. Il s'agirait, d'après l'hypothèse la plus récente et sans doute la plus séduisante, d'Adélaide, fille de la comtesse Hildegarde, fondatrice de l'église Sainte-Foy.

Le Moyen Age et la Renaissance entretiennent des relations de bon voisinage à Sélestat. Impossible d'y déceler la moindre solution de continuité dans leur coude à coude fraternel. On dirait même que le temps s'est arrêté dans cette ville douze fois séculaire. Les gens y marchent comme à pas feutrés, dirait-on, par crainte de rompre le rythme calme et réfléchi de la vieille ville. En plein midi, sur la place et dans les ruelles, caveaux et restaurants ont tôt fait de s'emplir on ne sait trop sous l'effet de quelle baguette magique. Sans bruit, sans hâte, à pied, à bicyclette, en voiture, Sélestadiens et visiteurs semblent avoir tout le temps du monde pour vaquer à leurs affaires. Ils évitent avec soin toute course fébrile, toute précipitation; chacun y va son bonhomme de train comme si de rien n'était. Quelle oasis de calme que cetté ville-carrefour, trait d'union entre les deux parties d'Alsace!

Le même charme envoûtant de la vie en ville ne manque point d'envahir le chercheur, dès qu'il est installé dans la Bibliothèque, où il est trop heureux de travailler à l'ombre de Beatus Rhenanus, ami d'Erasme de Rotterdam, de Josse Bade et de Lefèvre d'Etaples. Beatus Rhenanus (1485-1547), philologus, dont le buste occupe, comme il se doit, une place de choix à la Bibliothèque, ce mordu de l'érudition, des textes anciens et du livre bien fait, ce grand humaniste dévoré d'une immense curiosité intellectuelle, fit don de sa bibliothèque personnelle - 670 gros volumes - à la ville de Sélestat en 1547. Or, à l'époque de la Renaissance, rares étaient les bibliothèques publiques. Les savants devaient eux-mêmes, dans la mesure du possible, se procurer les volumes dont ils éprouvaient l'impérieux besoin. Qui plus est, la plupart des érudits de l'époque gardaient chez eux un fidèle famulus - celui de Beatus Rhenanus s'appelait Rodolphe Berz - étudiantsecrétaire, qui les aidait à écrire des lettres ou des préfaces, à vérifier des citations, à corriger des jeux d'épreuves, à inscrire les notes marginales du maître ou encore à trouver les plus belles éditions et les textes les plus rares. Doté d'une honnête fortune personnelle, Beatus Rhenanus put ainsi satisfaire son goût exquis et sa soif de connaissance exceptionnelle. Si des amis, comme le fameux imprimeur Froben, de Bâle, avec qui il collabora toute sa vie, lui donnèrent beaucoup de volumes, il en acheta cependant un plus grand nombre, car, dès l'âge de 15 ans, grâce à une maturité intellectuelle extraordinaire, il commença à réunir les plus belles éditions des textes anciens, les chefs-d'oeuvre des écrivains anciens: Cicéron, Virgile, Lucrèce, Suétone, Plaute, Stace, Pline le Jeune, les Pères de l'Eglise grecque et 


\section{4 / Renaissance and Reformation}

latine, Prosper, Lactance, saint Cyprien, saint Bède le Vénérable et saint Albert le Grand. Toute cette immense production, et j'en passe, on peut la trouver à Sélestat, avec la correspondance manuscrite de Beatus Rhenanus, soit 247 lettres à lui adressées et 7 envoyées par lui.

Les manuscrits de la bibliothèque de Sélestat, au nombre de 451 , portent sur les sujets les plus variés. Ils sont groupés sous une vingtaine de titres. Parmi les varia, j'y ai repéré et lu une lettre, fort intéressante, de Thomas Morus, aujourd'hui saint Thomas More (1478-1535). Quatre groupes de manuscrits sur vingt ont trait à l'histoire de Sélestat, de Strasbourg, des communes et de l'histoire générale de l'Alsace. Les manuscrits grecs (19) et les manuscrits des humanistes (21) occupent une place importante dans l'ensemble. Fait singulier, on y trouve un nombre égal de manuscrits des philosophes anciens (9), d'auteurs latins classiques (9) et de Pères de l'Eglise (9). Ajoutons deux manuscrits de médecins et de naturalistes (2), quatre de grammairiens et de lexicographes (4) et six d'annalistes, d'historiens et de voyageurs (6). De quoi occuper un régiment de candidats en mal de thèse pendant plusieurs décennies.

Cet aperçu, forcément sommaire et incomplet, ne comprend point une bonne demi-douzaine de disciplines, que je serais mal venu de passer sous silence et qui pourraient intéresser un autre solide régiment de spécialistes: l'Ecriture Sainte, la liturgie et le rituel, l'homilétique et la mystique, les règles monastiques, l'hagiographie et la mariologie, la théologie dogmatique et morale, le droit canon. Nombreux sont les manuscrits relatifs à ces divers domaines. On le voit, ce qui manque le plus à Sélestat, ce n'est certes pas le fonds, ce sont plutôt les chercheurs, les curieux intellectuels animés du feu sacré.

Mais à chacun son rayon. Comme je m'intéresse plus, affaire de formation et de tempérament, à l'Antiquité et à la Renaissance qu'au Moyen Age proprement dit, on me permettra d'insister davantage sur ces deux grandes époques de l'histoire de l'humanité de préférence à l'autre que je connais moins. Origène et Tertullien, saint Ambroise et saint Augustin, saint Athanase, saint Grégoire de Nazianze et saint Grégoire de Nysse, saint Benoît, saint Isidore et saint Grégoire le Grand, voire Cyrille, évêque d'Alexandrie ( $\mathrm{IX}^{\mathrm{e}}$ siècle); voilà autant d'écrivains de renom, dont la bibliothèque humaniste possède des manuscrits de leurs oeuvres. Ils datent principalement des $\mathrm{IX}^{\mathrm{e}}, \mathrm{X}^{\mathrm{e}}, \mathrm{XI}^{\mathrm{e}}$ et $\mathrm{XV}^{\mathrm{e}}$ siècles.

Parmi les manuscrits philosophiques des $\mathrm{XII}^{\mathrm{e}}$ et $\mathrm{XV}^{\mathrm{e}}$ siècles, quatre sont consacrés à Aristote et trois à Boèce; ce fait mériterait à lui seul un long commentaire. Les dictionnaires ou glossaires bilingues ne datent certes pas d'hier, à en juger par les manuscrits ayant trait aux voyages et aux annales. Ainsi j'ai pu consulter un glossaire latin-allemand, le texte latin étant déjà au $\mathrm{XI}^{\mathrm{e}}$ siècle accompagné d'une interprétation latine ou d'une traduction en langue germanique du $\mathrm{XI}^{\mathrm{e}}$ siècle; j'ai examiné un recueil latin-allemand de l'an 1100 et un itinéraire de voyage allemand-italien de 
Jean de Mandeville, daté de 1419. J'ai étudié aussi deux vocabulaires latinallemand de 1434 et 1450 respectivement: le Vocabularium modernum. . .et le Vocabularium super speculum grammatice. . .

Le bilinguisme gréco-latin se trouve, par exemple, dans les divers manuscrits de deux comédies d'Aristophane, les Nuées et Ploutos, qui semblent avoir été populaires aux $\mathrm{XV}^{\mathrm{e}}$ et $\mathrm{XVI}^{\mathrm{e}}$ siècles, à en juger du moins par le nombre d'exemplaires sur les rayons de la bibliothèque de Sélestat; la première est une satire de la nouvelle forme d'éducation en cours à Athènes pendant la guerre du Péloponèse, la seconde est une critique amusante du communisme décrit par Platon au livre $\mathrm{V}$ de sa République; la première répond aux réformateurs de l'enseignement à Athènes, la seconde, aux vues chimériques du jeune philosophe de l'Académie.

Les historiens de l'enseignement des humanités classiques auraient beaucoup de pain sur la planche s'ils passaient quelque temps à la bibliothèque de Sélestat. Ils y trouveraient, par exemple, des glossaires gréco-latins, des extraits d'auteurs grecs avec traduction latine en regard remontant au XV siècle, des copies d'inscriptions, des exercices gréco-latins, des prières en grec, un recueil grec de saint Jean Damascène et de saint Grégoire de Nysse, un recueil d'épigrammes, deux lexiques gréco-latins in $-2^{\circ}$ publiés à Bâle en 1563 et 1565 . Le Lexicon grecum S. Cyrilli episcopi Alexandrini (XII ${ }^{\mathrm{e}}$ siècle) et la Grammaire Grecque de Thomas Magister $\left(\mathrm{XV}^{\mathrm{e}}\right.$ siècle) mériteraient l'un et l'autre une étude particulière.

Il existe au moins 21 manuscrits des humanistes. Parmi les chefs de file, mentionnons le cardinal de Raguse avec son catalogue de livres grecs, Beatus Rhenanus, dont la correspondance contient une foule de renseignements précieux, Mélanchthon, qui a tant fait pour l'enseignement et l'étude du grec en Europe, Nicolas de Cuse (Alius Christianus. . .Dialogus de Deo abscondito), dont l'oeuvre exégétique et philosophique ne cesse d'être étudiée. Il existe une douzaine d'autres savants humanistes du XVI ${ }^{\mathrm{e}}$ siècle: auteurs, éditeurs, commentateurs, traducteurs, depuis longtemps tombés dans l'oubli ou injustement méconnus, dont la biographie et la bibliographie pourraient révéler quelques aspects nouveaux de l'humanisme.

La riche bibliothèque humaniste de Sélestat s'est constituée lentement au cours des siècles, comme bien l'on pense, par des donations successives de monastères et de savants. Les collections monastiques et les livres religieux en furent le principal noyau pendant un demi-siècle (1450-1500), c'est-à-dire de la fondation au début du $\mathrm{XVI}^{\mathrm{e}}$ siècle; ces volumes s'adressaient alors principalement aux religieux et aux élèves des Ecoles latines dirigées par eux. La fondation d'une bibliothèque paroissiale ou publique s'imposait d'autant plus que les manuscrits, par leur cherté et leur rareté, étaient accessibles à fort peu de gens; prêtres et laïques avides de culture ne pouvaient se les procurer pour approfondir et perfectionner leurs connaissances. L'Ecole latine de Sélestat, loin d'être une exception, avait besoin de livres; le maître d'école et ses élèves les réclamaient depuis 1440 


\section{6 / Renaissance and Reformation}

pour préparer leurs classes et cultiver leur esprit. C'est à partir de 1452 que fut constituée, grâce à une série de donations, la Bibliothèque paroissiale, d'où est issue la Bibliothèque humaniste.

Le premier legs fut celui de Jean de Westhus en 1452. Ce prêtre, fort cultivé et érudit, eut l'heureuse idée de faire venir à Sélestat Louis Dringenberg et de le placer à la direction de l'Ecole latine de la ville. A sa mort, en 1452, il fit don de sa librairie. Le chapelain Jean Fabri fit de même en 1470. Homme pieux et cultivé, poète et musicien, auteur de chants d'Eglise, il préparait ses sermons avec grand soin et se faisait un point d'honneur d'aider ses confrères dans leur ministère paroissial. On reconnaît aisément les livres de sa librairie qu'il légua par testament à la Bibliothèque par les remarques sur le temps ou sur les événements politiques et autres qu'il avait la manie d'inscrire sur la couverture à l'intérieur des volumes. Ils étaient aussi, comme ceux de Jean de Westhus, placés non pas sur des rayonnages mais plutôt sur des pupitres ou sur des tables, solidement munis de chaînes (libri catenati) pour les protéger contre le vol. En 1477, le célèbre maître de l'Ecole latine de Sélestat, Louis Dringenberg, fit à son tour cadeau de sa modeste librairie: deux manuscrits, contenant chacun le De consolatione de Boèce, un incunable, le Livre des Sentences, de Pierre Lombard. Cet écrivain, paraît-il, n'aurait jamais été traduit ni en anglais ni en français.

Fait pour le moins étrange, Jacques Wimpfeling, surnommé le praeceptor Germaniae, ne semble pas avoir pensé faire don de sa bibliothèque à sa ville natale; il ne lui légua même pas ses propres écrits, au nombre de 62 , que possède la Bibliothèque humaniste de Sélestat, grâce à des legs faits par Beatus Rhenanus et Alexandre Dorlan. Tout ce qu'elle reçut de lui, ce sont deux manuscrits, six incunables et trois imprimés du $\mathrm{XVI}^{\mathrm{e}}$ siècle. En revanche, Martin Ergersheim (1460-1535) fut beaucoup plus généreux. Prêtre fort cultivé, en relations constantes avec de nombreux humanistes et chercheur infatigable de belles éditions et de textes rares, il réussit à se constituer une précieuse et riche librairie. A sa mort, il la légua à son frère, Melchior, qui à son tour en fit don à la Bibliothèque de Sélestat, dont il avait été le dynamique prévôt. La donation Ergersheim est sans contredit la plus volumineuse jamais faite à la Bibliothèque paroissiale; elle est encore plus considérable que celle de Beatus Rhenanus, qui contenait près de 700 gros volumes.

Dès 1500 , et même un peu avant, la Bibliothèque possédait déjà de belles éditions, presque toutes italiennes, de la plupart des écrivains latins. Ces éditions de classiques, solidement reliées et placées à des tables où elles restaient enchaînées, servaient à l'usage quotidien des professeurs et de leurs meilleurs élèves. En voici une liste, forcément incomplète, avec la date et le lieu de l'édition: Cicéron (Venise, 1486, 1488), Horace (Venise, 1490), Virgile (Venise, 1492); Paris, s.d.), Ovide (Venise, 1491), Catulle, 
Properce, Tibulle (Venise, 1493), Quinte-Curce (Venise, 1502), Martial (Venise, 1503), Lucain (Venise, 1505), Stace (Venise, 1502).

Le chercheur trouvera à Sélestat les éditions principes de plusieurs Pères grecs et latins, des textes manuscrits des humanistes suivants: Petrus Hispanus, Jodocus Gallus, Johannes Wacker, Martin Dorpius, Henri de Gundelfingen, Sulpicius Verulanus, Jacques Taurellus, Guillaume Gisenheim, puis les ouvrages d'humanistes récents, tels que: Nicolaus Perottus, Franciscus Niger, Stephanus Fliscus, Augustinus Datus, Baptista Guarinus, Marius Philelphus. Aristote y occupe une place importante, à en juger par une vingtaine de ses traités, édités et commentés par Clichtovée ou Lefèvre d'Etaples, qui n'ont certes pas épargné les notes marginales, car elles figurent presque dans tous les volumes, ce qui montre l'emprise exercée par "le philosophe chrétien" sur Beatus et ses camarades d'études à Paris; son commentateur y était aussi tenu pour "le prince des philosophes." Tout cela, comme le fameux manuscrit d'Ovide (XIII ${ }^{\mathrm{e}}$ siècle), fait partie du fonds Rhenanus.

Un livre était un cadeau fort apprécié au $\mathrm{XVI}^{\mathrm{e}}$ siècle; un incunable et un manuscrit, bien sûr, l'étaient encore davantage. Les volumes coûtaient fort cher. Martin Ergersheim et Beatus Rhenanus, grâce à leur fortune personnelle, en achetèrent un grand nombre. Mais ils en reçurent aussi beaucoup d'amis et de collaborateurs en retour de services rendus, de conseils judicieux, ou encore à titre d'honoraires, les imprimeurs ayant alors l'habitude de donner un certain nombre d'exemplaires; ce qui conduisait à des échanges entre auteurs ou éditeurs. Ainsi Beatus Rhenanus inscrivait, avec un vif plaisir, sur la feuille de garde tantôt la date de l'achat ( 8 volumes en 1500, 14 en 1501, 35 en 1502), le montant dépensé et la formule de propriété: "Sum Beati Rhenani, nec muto dominum," tantôt la mention non moins célèbre: "dono dat, dono misit, muneri mittit." Quelle joie de lire ces phrases, qu'on croirait écrites d'hier! Plus profonde encore est la joie de travailler à Sélestat à l'ombre, pour ainsi dire, de savants imprimeurs et éditeurs, hellénistes ou latinistes, tels que: Froben, Josse Bade, Boniface Amerbach, Janus Cornarius, Jacques Wimpfeling, Willibald Pirckheimer, Beatus Arnoaldus, Cono (savant helléniste dominicain, dont les papiers personnels sont presque aussi précieux que les manuscrits), Lefèvre d'Etaples, Michel Hummelberg et Johannes Kierher.

Autant dire qu'un séjour de travail à la Bibliothèque humaniste de Sélestat vaut son pesant d'or. 